\title{
Nothnagel Syndrome: A rare case of Ischemic Brain Stem Syndrome
}

\author{
Ahmed Tanjimul Islam, ${ }^{1}$ Md. Kafil Uddin, ${ }^{2}$ Md. Ahmed Ali, ${ }^{2}$ Pijush Kumar Kundu, ${ }^{2}$ Md. Munzur Alahi ${ }^{3}$, \\ Mukul Kumar Sarkar ${ }^{3}$
}

\begin{abstract}
:
'Nothnagel Syndrome' is a brainstem syndrome which is often underdiagnosed by the physicians due to rarity of the disease and complex clinical presentation. We present a case of brainstem midbrain syndrome caused by ischemic stroke. The male hypertensive diabetic patient presented with sudden onset of diplopia, ptosis and gait imbalance. Right-sided gaze palsy with nystagmus and ataxic gait were the clinical findings. Brain imaging (MRI and DWI) showed high signal intensity at upper midbrain. The patient was finally diagnosed as ischemic stroke in the brainstem called Nothnagel syndrome. Up to now, there was no case reported as Nothnagel Syndrome due to ischemic stroke.
\end{abstract}

Key words: Ataxia, Brainstem Syndrome, Gaze palsy, Ischemia, Nystagmus.

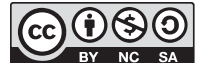

DOI: https://doi.org//0.3329/jom.v2lil.44I02

Copyright: (C) 2020 Khanom M. This is an open access article published under the Creative Commons Attribution-NonCommercial-NoDerivatives 4.0 International License, which permits use, distribution and reproduction in any medium, provided the original work is properly cited, is not changed in any way and it is not used for commercial purposes.

Received: 25 February, 20/9;

Accepted: 07 July, 2019

\section{Introduction:}

'Nothnagel Syndrome' is a rare clinical brainstem syndrome commonly involves the midbrain. Tumor is the most common aetiology for Nothnagel Syndrome. ${ }^{1}$ Paralysis of gaze, nystagmus, and ataxia are the diagnostic features. ${ }^{2}$ Anatomically the superior cerebellar peduncle of midbrain is the area involved for this rare brainstem syndrome. 1,2 Previously very few cases have been reported for this rare brainstem syndrome. ${ }^{3,4,5}$ Although prevalence of ischemic stroke is very high in South Asia region, no Nothnagel Syndrome case have been reported from this region. Again ischemic stroke was never been identified before as an aetiology for Nothnagel syndrome.

\section{Case report:}

A 60-year-old man presented with acute onset of double vision (diplopia) and difficulty in walking for 2 months. The diplopia was for horizontal eye movements, painless, bilateral, more marked on looking at right side. It was not associated with diurnal variation, eye pain, headache, vertigo or tinnitus. The difficulty in walking was sudden onset, not progressive \& tendency to fall sideways, which was more prominent when walking in a narrow passage. It was not associated with hemiplegia, sensory loss, speech disturbance or involuntary movements.

On clinical examination the patient is hypertensive (BP 170/ $90 \mathrm{~mm} \mathrm{Hg}$ ). On cranial nerve examination the patient had right sided incomplete ptosis, dilated pupil (Right), nystagmus in both eye, gaze palsy in both eye and diplopia for horizontal eye movement (false image outside) (Figure 1). Gait examination revealed cerebellar ataxia. Other systemic and neurological examination had no abnormality. Ophthalmoscopic examination (fundoscopy) revealed dot and blot hemorrhage in both retina.

Routine investigations revealed the patient was diabetic (RBS $18.4 \mathrm{mmol} / \mathrm{l}$ ). Initial imaging study with CT scan brain revealed tiny infarct in the thalamic region. Subsequent imaging study with MRI of brain showed hyper-intense lesion in upper midbrain and DWI (diffusion weighted

1. Medical Officer of Neurology, Rajshahi Medical College Hospital, Bangladesh

2. Associate Professor of Neurology, Rajshahi Medical College Hospital, Bangladesh

3. Assistant Professor of Neurology, Rajshahi Medical College Hospital, Bangladesh

Corresponding Author: Ahmed Tanjimul Islam, Medical Officer, Dept. of Neurology, Rajshahi Medical College Hospital, Bangladesh. Email: droveesomch@gmail.com, Phone: 8801554333026. 

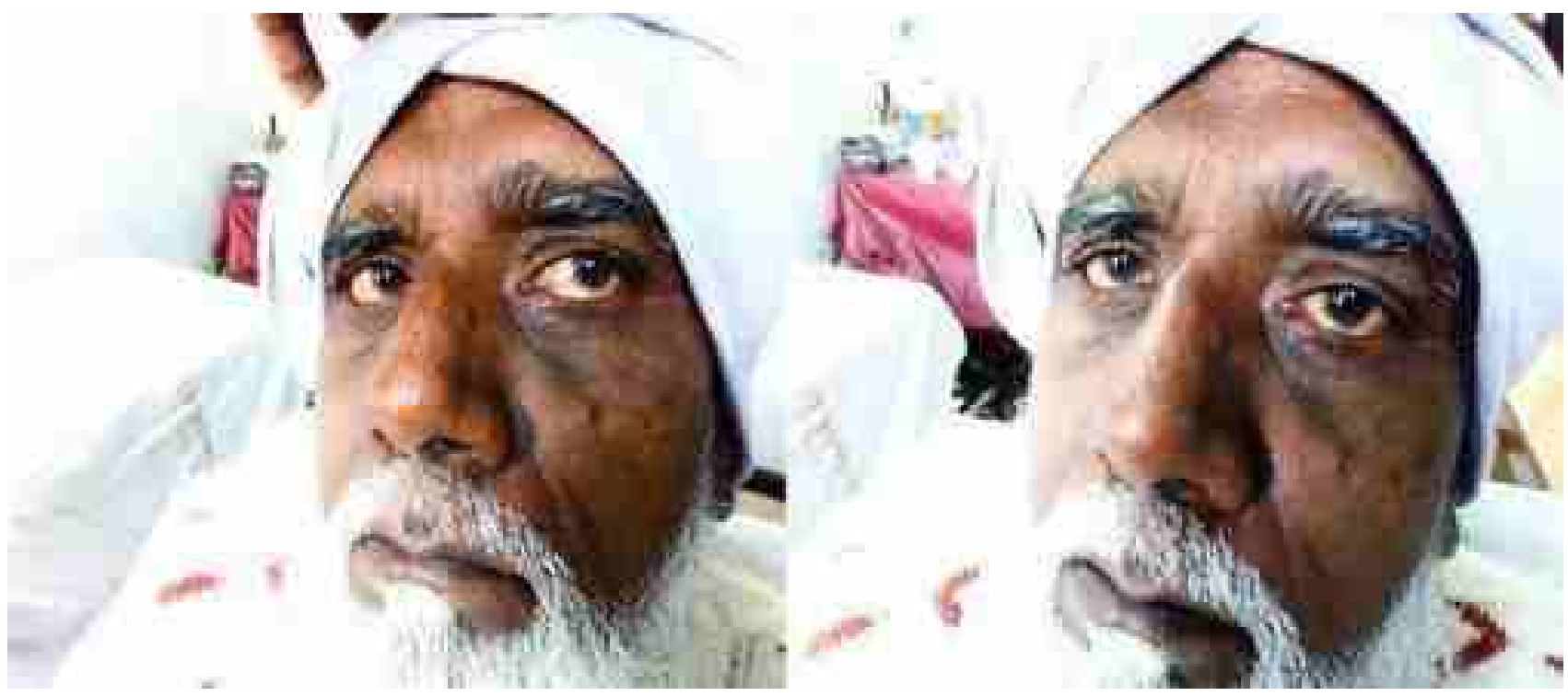

Figure 1: Normal gaze, looking at left (left image), gaze palsy with nystagmus in both eyes when looking at right. Both eyes failed to move to right (right image) (With patient's permission)

imaging) found restricted diffusion infarct in the midbrain region (Figure 2). Nothnagel Syndrome due to brainstem ischemic stroke was diagnosed. The patient was managed conservatively as ischemic stroke with co morbid conditions (hypertension and diabetes).

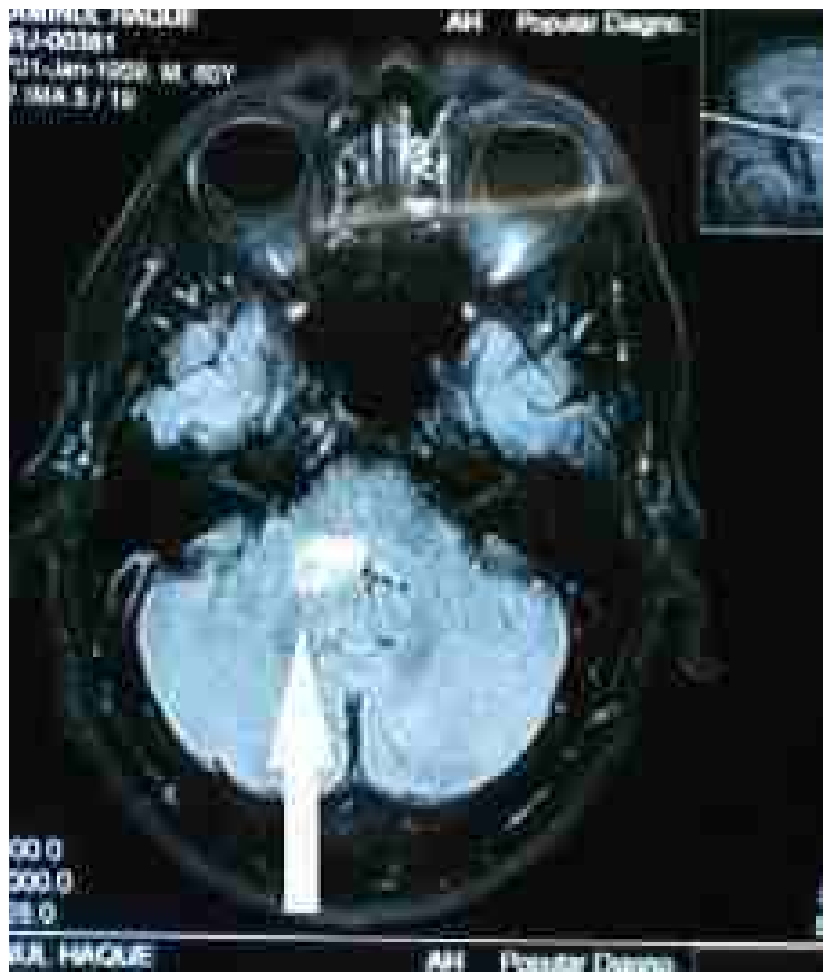

Figure 2: MRI of brain T2 showing hyper intense lesion in midbrain (infarction)

\section{Discussion:}

A famous German-Austrian physician, Hermann Nothnagel first described Nothnagel Syndrome in $1878 .{ }^{6}$ This brainstem syndrome is also known as dorsal midbrain syndrome. ${ }^{10}$ Structures commonly involved are superior cerebellar peduncle of midbrain and $3^{\text {rd }}$ nerve nucleus (oculomotor nucleus). Ipsilateral $3^{\text {rd }}$ nerve palsy, gaze palsy and cerebellar ataxia are the main components in Nothnagel Syndrome. 7,9 It can be used as a diagnostic criteria for this midbrain syndrome. ${ }^{7,8}$ Space occupying lesion usually the midbrain tumors are the common cause of Nothnagel Syndrome reported previously. ${ }^{8}$ Pathologically an association was found with midbrain glioma. ${ }^{9}$

The clinical presentations due to a pathology involving the quadri-geminal plate within the tectum of midbrain. Extension of the lesion to the $3^{\text {rd }}$ nerve nuclear and superior cerebellar peduncles causes this complex presentation. ${ }^{8,9}$ This syndrome is diagnosed by either clinical or radiological findings. Our case was diagnosed as Nothnagel Syndrome based on both clinical and radiological manifestations (MRI and DWI).

Posterior circulation (vertebro-basilar circulation) strokes are often associated with higher mortality and morbidity compared to other strokes. ${ }^{9}$ But there are better prognosis reported if the lesions are restricted to smaller vessels which causes minor changes and minimal area involvement. ${ }^{2,9}$ Management of this patient is done by conservatively (management of ischemic stroke) with physiotherapy (rehabilitation). 


\section{Conclusion:}

It is a diagnostic challenge for the clinicians to detect posterior circulation vascular lesion due to its complex presentation. In this rare case (Nothnagel Syndrome) the clinical presentation, examination and imaging finding all needs to be included to confirm the diagnosis. Patients presenting with gaze palsy and ataxia, a high index suspicion is needed to make an early correct diagnosis and treatment.

\section{Acknowledgement: Dr. MD Kafil Uddin.}

\section{Conflict of interest: None.}

\section{References:}

1. Schmidt D. Classical brain stem syndrome. Definitions and history. Ophthalmologe. 2000;97:411-7.

2. Liu GT, Crenner CW, Logigian EL, Charness ME, Samuels MA. Midbrain syndromes of Benedikt, Claude, and Nothnagel: setting the record straight. Neurology. 1992; 42: $1820-2$
3. Tanaka K, Sato T, Yamaguchi J. Stroke Rehabilitation for Nothnagel Syndrome : A Case Report. The Japanese Journal of Rehabilitation Medicine. 2007;44:280-285.

4. Derakhshan I, Sabouri-Deylami M, Kaufman B. Bilateral Nothnagel syndrome. Clinical and roentgenological observations. Stroke. 1980; 11:177-9.

5. Aizawa H, Morita K, Yamaguchi S, Sasaki N, Tobise K, Makita Y. A case of Nothnagel syndrome. Rinsho Shinkeigaku. 1996;36:889-91.

6. Nothnagel H. Corpora quadrigemina. In: Topische Diagnostik der Gehirnkrankheiten. A Hirschwald, 1879;204-220.

7. J. S. Kim, J. Kim. Pure midbrain infarction: clinical, radiologic, and pathophysiologic findings, Neurology, 2005;64:12271232 .

8. Moncayo J. Midbrain infarcts and hemorrhages. Frontiers of neurology and neuroscience. 30:158-61.

9. Ropper AH, Samuels MA, Klein JP. Adams and Victor's principles of neurology 10th ed. New York: McGraw-Hill Medical Pub. Division; 2014. 